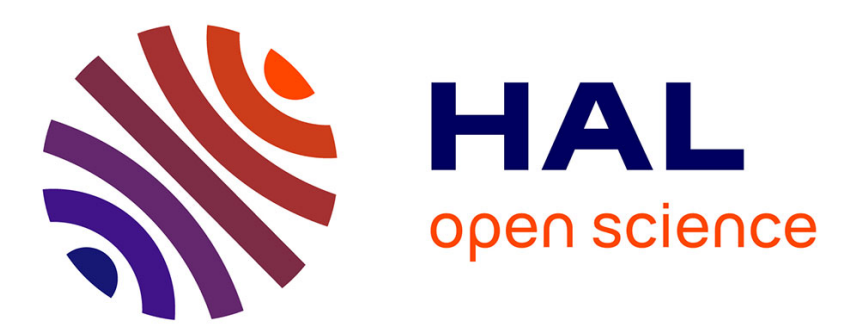

\title{
Inversion in indirect optimal control: constrained and unconstrained cases
}

François Chaplais, Nicolas Petit

\section{To cite this version:}

François Chaplais, Nicolas Petit. Inversion in indirect optimal control: constrained and unconstrained cases. 46th IEEE Conference on Decision and Control, Dec 2007, New Orleans, United States. pp.683689, 10.1109/CDC.2007.4434074 . hal-00501874

\section{HAL Id: hal-00501874 \\ https://hal-mines-paristech.archives-ouvertes.fr/hal-00501874}

Submitted on 12 Jul 2010

HAL is a multi-disciplinary open access archive for the deposit and dissemination of scientific research documents, whether they are published or not. The documents may come from teaching and research institutions in France or abroad, or from public or private research centers.
L'archive ouverte pluridisciplinaire HAL, est destinée au dépôt et à la diffusion de documents scientifiques de niveau recherche, publiés ou non, émanant des établissements d'enseignement et de recherche français ou étrangers, des laboratoires publics ou privés. 


\title{
Inversion in indirect optimal control: constrained and unconstrained cases
}

\author{
F. Chaplais and N. Petit
}

\begin{abstract}
This paper focuses on using non linear inversion in optimal control problems. This technique allows us to rewrite the stationarity conditions derived from the calculus of variations under a higher order form with a reduced number of variables. After a brief tutorial overview of the multiinput multi-output cases for which the cost functions have a positive definite Hessian with respect to control variables, we address the case of linear systems with a control affine cost to be minimized under input constraints. This is the main contribution of this paper. We study the switching function between singular and regular arcs and explain how higher order stationarity conditions can be obtained. An example from the literature (energy optimal trajectory for a car) is addressed.
\end{abstract}

\section{INTRODUCTION}

Lately, inversion has been used in direct methods of numerical optimal control (i.e. collocation). In such methods, coefficients are used to approximate both states and inputs [12] with basis functions. The numerical impact of the relative degree (as defined in [13]) of the output chosen to cast the optimal control problem into a nonlinear programming problem was emphasized in [18], [14]. Choosing outputs with maximum relative degrees is the key to efficient variable elimination that lowers the number of required coefficients (see for example [17], [7]). In differential equations, in constraints, and in cost functions, unnecessary variables are substituted with successive derivatives of the chosen outputs. When combined to a NLP solver (such as NPSOL [10] for instance), this can induce drastic speed-ups in numerical solving [5], [15], [19], [16], [1].

In [9], we focused on indirect methods (i.e. methods using adjoint variables) for numerically solving optimal control problems. In this framework, we explained how to use the geometric structure of the dynamics. In the single-input single-output (SISO) case (with an $n$-dimensional state), assuming the cost is quadratic in the control variables, we emphasized that $r$, the relative degree of the primal system, plays a role in the adjoint (dual) dynamics. The two-point boundary value problem (TPBVP) obtained from the calculus of variations can be rewritten by eliminating many variables. In fact, only $n-r$ variables are required. In the case of full feedback linearizability, the primal and adjoint dynamics take the form of a $2 n$-degree differential equation in a single variable: the linearizing output. More generally, we addressed

F. Chaplais is with the Centre Automatique et Systèmes, École Nationale Supérieure des Mines de Paris, 35, rue Saint-Honoré, 77305 Fontainebleau Cedex, France francois. chaplais@ensmp. fr.

N. Petit is with the Centre Automatique et Systèmes, École des Mines de Paris, 60, bd St Michel, 75272 Paris, Cedex 06, France nicolas.petiteensmp.fr. the general case of multi-inputs multi-outputs (MIMO) systems. Noting $m$ the number of inputs, and $r$ the total relative degree, we obtained a similar reduction of variables results. Numerous adjoint variables could be easily recovered once the optimal solution was known, providing direct insight into neighboring extremals and post-optimal analysis. Further, a strong positive impact (in terms of accuracy and CPU usage) of dealing with the obtained higher order representation of the TPBVP was underlined.

The results presented in [9] are quite general. Yet, several important questions remain to be explored. In particular, it often desired to deal with constrained inputs and non quadratic-like cost functionals. In such cases, calculus of variations may fail to give directly useable indications on the form of the optimal trajectories. In this paper, we aim at showing some first results that, despite the above mentioned restrictions, suggest that a similar approach can still be used.

The article is organized as follows. In Section II, we present in a tutorial manner the main result of [9]: while the system we wish to solve an optimal control problem for may only be partly feedback linearizable (i.e. may have a zero dynamics), it is possible to derive a $2 n$ dimensional necessary state space form equation for the primal and adjoint dynamics using a reduced number of variables $(m+2(n-r))$. Adjoint states corresponding to the linearizable part of the dynamics are explicitly computed and eliminated from stationarity conditions. This is summarized in Theorem 2. In this case, the main hypothesis are that the system has a control affine dynamics and that the cost functional has a positive definite Hessian with respect to the control variables. Further, in Section III, we consider the case of linear systems with a cost functional that is affine in the control variable. The elimination technique is rather different. In this study, we limit ourselves to single-input single-output (SISO) systems. A key element in the derivation of a higher order form of the stationarity conditions is the switching functions that define singular and regular arcs. We prove a general result (Theorem 3) which stresses that, along singular arcs, the order of differentiation that appears is lower than in the regular case. This is the main contribution of the paper. Exploiting this observed structure surely requires dedicated numerical tools. These are out of the scope of this paper. Rather, in Section IV, we study an example from the literature and show an interesting property. The example under consideration is a trajectory optimisation problem for an energy-efficient car (see [11] for a complete exposition of the problem statement and solutions). Following our method, we deduce in a very straightforward manner that, along singular arcs, the velocity 
of the car is constant.

\section{The UnCONSTRAIned MIMO CASE}

In this section, we present, in a (hopefully) tutorial manner, the main result of [9] (which is rather technical). Whenever necessary, the reader should directly refer to [9] for precise justification of several statements. The presentation is rather informal and is focused on the inversion procedure.

\section{A. Problem statement and stationarity conditions}

Consider a dynamical control system with multiple inputs that is put, after a suitable change of coordinates, into the following normal form consisting of $m$ chains of integrators of lenghts $\left(m_{i}\right)_{i=1, \ldots, m}$ and a zero dynamics [13] with dimension $q$.

$$
\begin{gathered}
\frac{d x_{i}^{1}}{d t}=x_{i}^{2}, \ldots, \frac{d x_{i}^{r_{i}}}{d t}=L_{f}^{r_{i}} h_{i}+\sum_{j=1}^{m} a_{i j} u_{j} \triangleq v_{i} \\
\frac{d \eta_{j}}{d t}=\alpha^{j}(X)+\sum_{i=1}^{m} \beta_{i}^{j}(X) v_{i}=\mathcal{B}_{j}(X, v)
\end{gathered}
$$

where $\alpha^{j}$ and $\beta_{i}^{j}$ are smooth $\mathbb{R}^{n} \rightarrow \mathbb{R}$ mappings, for all $j=1, \ldots, q$, and $i=1, \ldots, m . X$ is the vector of all state variables and $v$ is a state feedback (defined in (1)) of the linearizing outputs $\left(x_{i}\right)_{i=1, \ldots, m}$ and their derivatives.

We wish to solve an unconstrained optimal control problem where it is desired to minimize the cost function

$$
\int_{0}^{T} \mathcal{L}(X(t), v(t)) d t
$$

where $\mathcal{L}$ is a smooth mapping. This minimization problem can arise from a similar problem in original coordinates (before the normal form is used). In that case, transformations on the state and the control impact on the definition of $\mathcal{L}$.

An important feature of this setup is that, if the cost function has a positive definite Hessian with respect to the control variables $\left(u_{i}\right)_{i=1, \ldots, m}$, then this is also true with respect to the control variables $\left(v_{i}\right)_{i=1, \ldots, m}$ after the change of coordinates and the state feedback. In the following, we assume that this property holds.

Following the lines of Pontryagin minimum principle (PMP) (see e.g. [6]), we obtain an Hamiltonian with the following special structure

$$
\begin{aligned}
H=\mathcal{L}(X, v)+\sum_{i=1}^{m} \sum_{j=1}^{r_{i}-1} & \lambda_{i}^{j} x_{i}^{j+1} \\
& \quad+\sum_{i=1}^{m} \lambda_{i}^{r_{i}} v_{i}+\sum_{j=1}^{q} \mu_{j} \mathcal{B}_{j}(X, v)
\end{aligned}
$$

where $\lambda \triangleq\left(\lambda_{1}^{1}, \ldots, \lambda_{1}^{r_{1}}, \ldots, \lambda_{m}^{1}, \ldots, \lambda_{m}^{r_{m}}\right)^{T}$ represents the adjoint states related to the chains of integrators (1) and $\mu \triangleq\left(\mu_{1}, \ldots, \mu_{q}\right)^{T}$ corresponds to the zero dynamics (2). Exploiting this structure, we can readily realize that the stationarity conditions bearing on the adjoint variables write under the particular cascaded form

$$
\begin{gathered}
\frac{d \lambda_{i}^{1}}{d t}=-\frac{\partial \mathcal{L}}{\partial x_{i}^{1}}-\sum_{j=1}^{q} \mu_{j} \frac{\partial \mathcal{B}_{j}}{\partial x_{i}^{1}}, \quad i=1 \ldots p \\
\frac{d \lambda_{i}^{j}}{d t}=-\lambda_{i}^{j-1}-\frac{\partial \mathcal{L}}{\partial x_{i}^{j}}-\sum_{k=1}^{q} \mu_{k} \frac{\partial \mathcal{B}_{k}}{\partial x_{i}^{j}}, \\
\frac{d \mu_{j}}{d t}=-\frac{\partial \mathcal{L}}{\partial \eta_{j}}-\sum_{k=1}^{q} \mu_{k} \frac{\partial \mathcal{B}_{k}}{\partial \eta_{j}}, \quad j=1 \ldots q, j=2 \ldots r_{i}
\end{gathered}
$$

Further, PMP states that, along normal optimal trajectories, the Hamiltonian is minimum with respect to $v$. In the absence of constraints on $v$, this translates as

$$
\frac{\partial H}{\partial v_{i}}=\frac{\partial \mathcal{L}}{\partial v_{i}}+\lambda_{i}^{r_{i}}+\sum_{j=1}^{q} \mu_{j} \beta_{i}^{j}(X)=0
$$

\section{B. An implicit differential system}

Two important facts are to be noted here:

- Since (4) is identically zero all along the optimal trajectory, its derivatives with respect to time are also zero.

- The cascade dynamics and its "mirror" on the adjoint state dynamics makes the adjoint states progressively disappear as we differentiate (4).

After some computations, these properties yield the following lemma

Lemma 1: Let $i \in\{1 \ldots m\}$ such that $r_{i} \geq 2$. For $j=$ $1 \ldots r_{i}-1$, there exists a function $G_{i}^{j}$ from $\mathbb{R}^{2 n-r+m j}$ to $\mathbb{R}$ such that

$$
\begin{aligned}
\frac{d^{j}}{d t^{j}} \frac{\partial H}{\partial v_{i}}=\sum_{k=1}^{m} & \frac{\partial^{2} \mathcal{L}}{\partial v_{i} \partial v_{k}} x_{k}^{\left(r_{k}+j\right)}+(-1)^{j} \lambda_{i}^{r_{i}-j} \\
& +G_{i}^{j}\left(\eta, \mu, \ldots, x_{l} \ldots x_{l}^{\left(r_{l}+j-1\right)}, \ldots\right)
\end{aligned}
$$

where $l$ is a running index ranging from 1 to $m$.

The proof mainly uses standard differential calculus. More precisely, it is organized around the following two points:

- The coefficients of the highest order derivatives of the linearizing outputs can be successively and independently derived (each of these coefficients is a component of the Hessian of the cost with respect to the control variables).

- The reverse cascade structure of the dynamics of the adjoint states implies that, as we differentiate (5), we decrease the indices of the adjoint states that appear in the equation. Since the dynamics of $\lambda_{i}^{1}$ does not involve any $\lambda_{i}^{j}$, differentiating the last (5) (i.e. for $j=r_{i}-1$ ) leads to a set of equations where the adjoint states corresponding to the linearizing outputs and their derivatives have disappeared. This is stated in the following theorem. 
Theorem 1: For $i=1 \ldots m$, there exists a function $G_{i}$ from $\mathbb{R}^{2 n-r+m r_{i}}$ to $\mathbb{R}$

$$
G_{i}\left(\eta, \mu, \ldots, x_{l} \ldots x_{l}^{\left(r_{l}+r_{i}-1\right)}, \ldots\right)
$$

where $l$ is a running index ranging from 1 to $m$, such that

$$
\begin{aligned}
\sum_{k=1}^{m} \frac{\partial^{2} \mathcal{L}}{\partial v_{i} \partial v_{k}} & x_{k}^{\left(r_{k}+r_{i}\right)} \\
& +G_{i}\left(\eta, \mu, \ldots, x_{l} \ldots x_{l}^{\left(r_{l}+r_{i}-1\right)}, \ldots\right)=0
\end{aligned}
$$

Together with (2) and (3), these equations form a set of differential equations on $x_{i}, i=1, \ldots, m, \eta$ and $\mu$ from which the $\lambda_{i}^{j}, i=1, \ldots, m, j=1, \ldots, r_{m}$, have been completely eliminated.

Theorem 1 states that an implicit differential system that must be satisfied by the linearizing outputs can be obtained. Turning this system into an explicit form is not straightforward in the MIMO case (the SISO case is much simpler and is addressed in [8]). Computing the Jacobian of the system with respect to the highest derivatives of the linearizing outputs, and assuming that we have reordered the linearizing outputs in the decreasing length of their integration chains, some sparsity appears. Zero terms appear everywhere except on the first lines which corresponds to the variables with the highest derivatives (and the longest chain length). In practice, to turn the system into an explicit form, we have to perform a recursive differential elimination of some derivatives.

\section{An explicit differential system}

The algorithm that we use to transform the implicit system (6) into an explicit one is outlined below. To simplify things, we assume that all the lengths of the integrators chain are different. Again, the general case is addressed in [9].

We start with the last line of the differential system, i.e.

$$
\begin{aligned}
& \sum_{k=1}^{m} \frac{\partial^{2} \mathcal{L}}{\partial v_{m} \partial v_{k}} x_{k}^{\left(r_{k}+r_{m}\right)} \\
& \quad+G_{m}\left(\eta, \mu, \ldots, x_{l} \ldots x_{l}^{\left(r_{l}+r_{m}-1\right)}, \ldots\right)=0
\end{aligned}
$$

where $r_{m}$ is the shortest length of integrator chains. Since $\frac{\partial^{2} \mathcal{L}}{\partial^{2} v_{m}}$ is invertible (it is on the diagonal of the Hessian of the cost), we obtain that $x_{m}^{\left(2 r_{m}\right)}$ is a function of $x_{m} \ldots x_{m}^{\left(2 r_{m}-1\right)}$, $x_{m-1} \ldots x_{m-1}^{\left(r_{m-1}+r_{m}-1\right)}$ and various derivatives of the other linearizing outputs and, of course, $\eta$ and $\mu$. Observe that $r_{m-1}+r_{m}-1<2 r_{m-1}$. Then, repeated differentiations of (7) give rise to equations of the form

$$
\begin{aligned}
\sum_{k=1}^{m} \frac{\partial^{2} \mathcal{L}}{\partial v_{m} \partial v_{k}} x_{k}^{\left(r_{k}+r_{m}+j\right)} & \\
& +\tilde{G}_{m}\left(\eta, \mu, \ldots, x_{l} \ldots x_{l}^{\left(r_{l}+r_{m}-1+j\right)}\right. \\
& \left.\ldots, x_{m} \ldots x_{m}^{\left(2 r_{m}-1\right)}\right)=0
\end{aligned}
$$

One can notice that the expression of the derivative of (7) involves higher derivatives of the linearizing outputs except for the derivatives of $x_{m}$ in $\tilde{G}_{m}$ whose orders are not increased. Observe that, for $j=r_{m-1}-r_{m}$, we have an equation where the derivatives of $x_{m-1}$ and $x_{m}$ (in the summation at the left) are of order $2 r_{m-1}$ and $r_{m}+r_{m-1}$, and that $\tilde{G}_{m}$ does not involve derivatives of $x_{m}$ of an order greater or equal to $2 r_{m}-1$.

Now, look at the penultimate line of equations in (6). This equation has a very similar structure, except higher order derivatives of $x_{m}$ appear. Yet, these derivatives can be rewritten as a function of $x_{m} \ldots x_{m}^{\left(2 r_{m}-1\right)}$ and various derivatives of the other linearizing outputs (various derivatives of $\eta$ and $\mu$ are rewritten using the zero dynamics and its related adjoint state dynamics). Putting this reformulated equation together with the adequately rewritten $\left(r_{m-1}-r_{m}\right)^{t h}$ derivative of the last line gives rise to a linear system of two equations in the unknowns $x_{m-1}^{\left(2 r_{m-1}\right)}$ and $x_{m}^{\left(2 r_{m}\right)}$. The corresponding matrix is diagonally extracted from the Hessian matrix of the cost function with respect to the controls. The right handside involves derivatives of $x_{m-1}$ (resp. $x_{m}$ ) up to the order $2 r_{m-1}-1$ (resp. $2 r_{m}-1$ ). Solving this system yields an expression of $x_{m-1}^{\left(2 r_{m-1}\right)}$ and $x_{m}^{\left(2 r_{m}\right)}$. This expression can be used to eliminate higher order derivatives of $x_{m-1}$ and $x_{m}$ throughout the remaining computations.

Following the same computational method, we can differentiate the last two modified lines of the differential system until, together with the line before the penultimate one, we obtain a system of three equations with respect to three high order derivatives of the last three linearizing outputs, and so forth.

Details of this algorithm for two and three inputs, as well as the general case, are presented in [9]. Induction of this method up to the first line of the differential system leads to the following theorem:

Theorem 2: For $i=1, \ldots, m$, there exist functions $G_{i}\left(\eta, \mu, x_{1} \ldots x_{1}^{\left(2 r_{1}-1\right)}, \ldots, x_{m} \ldots x_{m}^{\left(2 r_{m}-1\right)}\right)$ such that

$$
\begin{aligned}
x_{i}^{\left(2 r_{i}\right)} & = \\
& G_{i}\left(\eta, \mu, x_{1} \ldots x_{1}^{\left(2 r_{1}-1\right)}, \ldots, x_{m} \ldots x_{m}^{\left(2 r_{m}-1\right)}\right)
\end{aligned}
$$

Now consider, (8) for $i=1, \ldots, m$, along with the state equations on $\eta$ and $\mu$ (respectively, (2) and (3)). This set of equations forms a higher order explicit differential system which must be satisfied by the linearizing outputs (and $(\eta, \mu))$.

Eventually, a two-point boundary value problem can be obtained as follows. The initial conditions on the state provide initial conditions. Then, through (4) and (5), the conditions on the final values of the adjoint sates provide final conditions on the variables of (8). In summary, we obtain a higher order two boundary value problem using a limited number of variables. This number is $m+2(n-$ $\left.\sum_{i=1}^{m} r_{i}\right)$.

Once (8), (2) and (3) are solved, $\eta$ and the derivatives of the linearizing outputs provide the values of the state; equations (4) and (5), together with the knowledge of $\mu$, give the value of the adjoint state; finally, solving equations (1) with respect to the controls $u_{j}$ gives the value of the optimal control. 


\section{Impact on numerical resolution}

Of course, the differential system (8) possesses an equivalent first order state space form. A theoretical result by Ascher-et-al [3], [4, pp. 220-222] shows that using a collocation method on a higher-order equations is more accurate than using it on its corresponding first-order form. More precisely, while similar accuracy is obtained at the mesh points, the higher order representation yields a superior accuracy between the mesh points. Using the colnew collocation software package by Ascher-et-al (available through the open-source scientific environment Scilab), we have experimentally compared the accuracy of collocation on a first order state space model and collocation on the derived higher order model. A benchmark problem was used for which the optimal control is known analytically. This study confirms the superiority of the higher order method in between the mesh points (see [9]).

Another experiment (on a problem where no analytical solution is known) compares the computational burden of the colnew package for first order and higher order models. Results are consistent with experiments conducted with the direct method (i.e. not using adjoint variables) software package NTG [14]. For similar computed constraint violations, the higher order methods are clearly more efficient in terms of computational burden (see [9] for details).

\section{A CONSTRAINED SISO PROBLEM WITH LINEAR DYNAMICS AND A COST WHICH IS AFFINE IN THE CONTROL}

\section{A. Problem statement}

We now focus on a class of optimal control problems that give rise to singular arcs. The preceding elimination procedure can not be used anymore because the Hessian of the cost function with respect to control variables is not definite positive. Yet, it inspires an interestingly formally similar approach. We present here preliminary results that are limited to SISO systems. For sake of simplicity of exposition, the dynamics is assumed linear, but, as appears in Section IV, this is not a real limitation. Future research will focus on MIMO nonlinear cases.

1) Optimal control problem statement: We consider a linear SISO system and an optimal control problem where the integral cost is affine with respect to the control $u$. A static input constraint $u \in\left[u_{\min }, u_{\max }\right]$ is imposed. A linear basis change in the state space allows us to assume that the dynamics are under a controllable normal form, i.e. the dynamics can be written as

$$
\left\{\begin{aligned}
\frac{d x_{1}}{d t} & =x_{2} \\
& \vdots \\
\frac{d x_{i}}{d t} & =x_{i+1} \\
\vdots & \\
\frac{d x_{n}}{d t} & =u+\sum_{i=1}^{i=n} c_{i} x_{i}
\end{aligned}\right.
$$

Here, we do not perform any feedback transformation on the last line of (9) because the constraints on the control would then be transformed into state constraints. This would result in possible discontinuities of the adjoint states at switching times from regular to singular arcs (see below), which we wish to avoid.

We assume that the integral cost $\int_{0}^{T} L(X, u, t) d t$ to be minimized is of the form

$$
\int_{0}^{T} L(X, u, t) d t=\int_{0}^{T}(a(X) u+b(X)) d t .
$$

More generally, a terminal cost and final point constraints may be considered without too much effort.

2) The Pontryagin minimum principle (PMP): Consider the Hamiltonian $H(\lambda, X, u)$ defined by

$$
\begin{aligned}
& H(\lambda, x, u)= \\
& \sum_{i=1}^{i=n-1} \lambda_{i} x_{i+1}+\lambda_{n}\left(\sum_{i=1}^{i=n} c_{i} x_{i}+u\right)+a(X) u+b(X)
\end{aligned}
$$

In this case, the PMP states that, if an optimal control exists, then there exists an adjoint state $\lambda(t)$ which satisfies the socalled adjoint equations

$$
\left\{\begin{aligned}
\frac{d \lambda_{1}}{d t} & =-\lambda_{n} c_{1}-\frac{\partial a}{\partial x_{1}} u_{o p t}-\frac{\partial b}{\partial x_{1}} \\
& \vdots \\
\frac{d \lambda_{i}}{d t} & =-\lambda_{i-1}-\lambda_{n} c_{i}-\frac{\partial a}{\partial x_{i}} u_{o p t}-\frac{\partial b}{\partial x_{i}} \\
& \vdots \\
\frac{d \lambda_{n}}{d t} & =-\lambda_{n-1}-\lambda_{n} c_{n}-\frac{\partial a}{\partial x_{n}} u_{o p t}-\frac{\partial b}{\partial x_{n}}
\end{aligned}\right.
$$

where $u_{o p t}$ is the optimal control. Additionally, the final values of the adjoint states satisfy conditions determined by the terminal cost and the final point state constraints. Finally, the optimal control $u_{\text {opt }}$ minimizes the Hamiltonian $H(\lambda(t), X(t), u)$ over the possible values $\left[u_{\min }, u_{\max }\right]$.

3) The switching function between singular and regular arcs: One can easily see that minimizing the Hamiltonian is equivalent to minimizing $\left(\lambda_{n}(t)+a(X(t))\right) u$. Then, $\frac{\partial H}{\partial u}=$ $\lambda_{n}(t)+a(X(t))$ is called the switching function. If it is non zero, then either $u_{o p t}=u_{\text {min }}$ or $u_{o p t}=u_{\max }$ depending on the sign of the switching function. When the switching function is non zero, the portions of the optimal trajectory 
where this condition is satisfied are called regular arcs. By contrast, portions of the optimal trajectory where the switching function is identically zero are called singular arcs.

We concentrate our attention on singular arcs which are closed intervals with a non empty interior. On such interval, the following simple equation holds

$$
\frac{\partial H}{\partial u}=\lambda_{n}(t)+a(X(t))=0
$$

Notations: Considering the structure of (9), we denote by $x$ the first component $x_{1}$ of the state $X$. We have, for $i=$ $1 \ldots n, x_{i}=x^{(i-1)}$ and $x^{(n)}=u+\sum_{i=1}^{i=n} c_{i} x^{(i-1)}$. In the following, whenever it is possible, we shall write $u$ instead of $u_{\text {opt }}$.

\section{B. Derivatives of the switching function}

We are going to differentiate equation (12) to derive a higher order differential equation on $x$ which determines the primal and adjoint states, and the optimal control.

1) First derivative: Since (12) holds along the singular arc, its derivative with respect to time is also zero. Straightforward computations along with the use of (11) and (12) lead to

Lemma 2: The first derivative of the switching function satisfies

$$
\begin{aligned}
& \frac{d}{d t}\left(\frac{\partial H}{\partial u}\right)=-\lambda_{n-1}+a(X) c_{n}-\frac{\partial b}{\partial x^{(n-1)}}(X) \\
& \quad+\sum_{i=0}^{i=n-2} \frac{\partial a}{\partial x^{(i)}} x^{(i+1)}+\frac{\partial a}{\partial x^{(n-1)}} \sum_{i=1}^{i=n} c_{i} x^{(i-1)}=0
\end{aligned}
$$

with $X=\left(x, \ldots, x^{(n-1)}\right)$.

Proof: From (11), we get

$$
\frac{d \lambda_{n}}{d t}=-\lambda_{n-1}-\lambda_{n} c_{n}-\frac{\partial a}{\partial x^{(n-1)}} u-\frac{\partial b}{\partial x^{(n-1)}}
$$

On the other hand,

$$
\begin{aligned}
\frac{d}{d t}(a(X))=\sum_{i=0}^{i=n-2} & \frac{\partial a}{\partial x^{(i)}} x^{(i+1)} \\
& +\frac{\partial a}{\partial x^{(n-1)}}\left(u+\sum_{i=1}^{i=n} c_{i} x^{(i-1)}\right)
\end{aligned}
$$

Summing up (14) and (15) make $u$ disappear. Substituting $\lambda_{n}=-a(X)$ (equation (12)) in this sum, we obtain (13).

Remark: Equations (12) and (13) are involving variables which are continuous at the boundary of the singular arc. Hence, both must hold at the corresponding boundary of the neighboring regular arc.

2) Further derivatives:
Lemma 3: Let $d(X)$ be defined by

$$
\begin{array}{r}
d(X)=\frac{\partial a}{\partial x^{(n-2)}}+2 \frac{\partial a}{\partial x^{(n-1)}} c_{n}-\frac{\partial^{2} b}{\left(\partial x^{(n-1)}\right)^{2}} \\
+\sum_{i=0}^{i=n-2} \frac{\partial^{2} a}{\partial x^{(i)} \partial x^{(n-1)}} x^{(i+1)}+\frac{\partial a}{\partial x^{n-2}} \\
+\frac{\partial^{2} a}{\left(\partial x^{(n-1)}\right)^{2}} \sum_{i=1}^{i=n} c_{i} x^{(i-1)}
\end{array}
$$

We assume that $d(X)$ does not vanish along the singular arc under study. Then, for $i=2, \ldots, n-1$, there exists $e_{i}\left(x, \ldots, x^{(n+i-3)}\right)$ such that, along this singular arc,

$$
\begin{aligned}
\frac{d^{i}}{d t^{i}}\left(\frac{\partial H}{\partial u}\right)=(-1)^{i} \lambda_{n-i} & +d(X) x^{(n+i-2)} \\
& +e_{i}\left(x, \ldots, x^{(n+i-3)}\right)=0
\end{aligned}
$$

Proof: We start with $i=2$ and differentiate (13). Observing that $\lambda_{n}=-a(X)$, we have

$$
\begin{aligned}
\frac{d\left(-\lambda_{n-1}\right)}{d t} & = \\
& \lambda_{n-2}-a(X) c_{n-1}+\frac{\partial a}{\partial x^{(n-2)}} u+\frac{\partial b}{\partial x^{(n-2)}}
\end{aligned}
$$

Substituting $u=x^{(n)}-\sum_{i=1}^{i=n} c_{i} x^{(i-1)}$, we see that there exists $\alpha_{1}(X)$ such that

$$
\frac{d\left(-\lambda_{n-1}\right)}{d t}=\lambda_{n-2}+\frac{\partial a}{\partial x^{(n-2)}} x^{(n)}+\alpha_{1}(X)
$$

One can easily observe that there exists $\alpha_{2}(X)$ such that

$$
\frac{d}{d t}\left(a(X) c_{n}\right)=\frac{\partial a}{\partial x^{(n-1)}} x^{(n)} c_{n}+\alpha_{2}(X)
$$

The derivative of the next term in (13) is such that there exists $\alpha_{3}(X)$ with

$$
\frac{d}{d t}\left(-\frac{\partial b}{\partial x^{(n-1)}}(X)\right)=-\frac{\partial^{2} b}{\left(\partial x^{(n-1)}\right)^{2}} x^{(n)}+\alpha_{3}(X)
$$

There also exists $\alpha_{4}(X)$ such that the derivative of the next term in (13) satisfies

$$
\begin{aligned}
\frac{d}{d t}\left(\sum_{i=0}^{i=n-2} \frac{\partial a}{\partial x^{(i)}} x^{(i+1)}\right) & = \\
& \left(\sum_{i=0}^{i=n-2} \frac{\partial^{2} a}{\partial x^{(i)} \partial x^{(n-1)}} x^{(i+1)}\right) x^{(n)} \\
& +\frac{\partial a}{\partial x^{(n-2)}} x^{(n)}+\alpha_{4}(X)
\end{aligned}
$$

Finally, there exists $\alpha_{5}(X)$ such that

$$
\begin{aligned}
& \frac{d}{d t}\left(\frac{\partial a}{\partial x^{(n-1)}} \sum_{i=1}^{i=n} c_{i} x^{(i-1)}\right)= \\
& \left(\frac{\partial^{2} a}{\partial\left(x^{(n-1)}\right)^{2}} \sum_{i=1}^{i=n} c_{i} x^{(i-1)}\right) x^{(n)} \\
& \quad+\frac{\partial a}{\partial x^{(n-1)}} c_{n} x^{(n)}+\alpha_{5}(X)
\end{aligned}
$$


Summing (18), (19), (20), (21) and (22), eventually gives (17) for $i=2$ with $d(X)$ defined by (16).

To show the induction of (17) from $i$ to $i+1$, we need further differentiations. First, we observe that $x^{(n+i-2)}$ is differentiable because $d(X)$ is non zero. The other variables in (17) are differentiable since they are either an adjoint state or a lower order derivative of $x$. The induction is then proved by differentiating (17) with respect to time. We use the adjoint equations (11), the equality $u=x^{(n)}-\sum_{i=1}^{i=n} c_{i} x^{(i-1)}$, and the expression of $\lambda_{n}$ which is given by (12). Finally, the differentiation of $d(X) x^{(n+i-2)}+e_{i}\left(x, \ldots, x^{(n+i-3)}\right)$ gives terms of the form $d(X) x^{(n+i-2+1)}+e_{i+1}\left(x, \ldots, x^{(n+i-2)}\right)$ because $n+i-2 \geq n$.

Remark: On a singular arc, $X$ is bounded because it is continuous on a closed interval; $u$ is bounded by definition of the constraint; hence $x^{(n)}$ is bounded on the arc. Since (17) holds for $i=2$, we see that $d(X)$ is zero if and only if $\lambda_{n-2}+e(X)$ is zero. This may happen in particular cases: for instance, if the integral cost is linear time invariant with respect to $(X, u)$, then $a$ is a constant and the second derivatives of $b$ with respect to $x$ are zero; therefore $d(X)$ is identically zero in this case. This case is detailed in the appendix. In what follows, we shall assume that $d(X)$ does not vanish on the singular arc. As we shall see in the following, this will allows to derive a higher order differential equation satisfied by $x$.

\section{A higher order differential equation in $x$ along singular} $\operatorname{arcs}$

Theorem 3: We assume $n \geq 3$. If $d(X)$ does not vanish along the singular arc, then there exists a function $e_{n}\left(x, \ldots, x^{(2 n-3)}\right)$ such that

$$
\begin{aligned}
& \frac{d^{n}}{d t^{n}}\left(\frac{\partial H}{\partial u}\right)= \\
& d(X) x^{(2 n-2)}+e_{n}\left(x, \ldots, x^{(2 n-3)}\right)=0
\end{aligned}
$$

Proof: Essentially, to derive this result, we differentiate (17) with respect to time for $i=n-1$. The main point is that the expression $\frac{d \lambda_{1}}{d t}$ does not involve any adjoint state other than $\lambda_{n}$. Indeed, if we consider the first line of (11), since $\lambda_{n}=-a(X)$ and $u=x^{(n)}-\sum_{i=1}^{i=n} c_{i} x^{(i-1)}$, we see that $\frac{d \lambda_{1}}{d t}$ is a function of $\left(x, \ldots, x^{(n)}\right)$ and, more generally, since $n \geq 3$, it is also a function of $\left(x, \ldots, x^{(2 n-3)}\right)$. Following the lines of the induction in the proof of lemma 3 , we see that the derivative of $d(X) x^{(2 n-3)}+e_{n-1}\left(x, \ldots, x^{(2 n-4)}\right)$ is of the form $d(X) e^{(2 n-2)}+\tilde{e}_{n}\left(x, \ldots, x^{(2 n-3)}\right)$. Summing the two expressions proves (23).

Boundary values: For $i=1, \ldots, n-1, x^{(i)}$ is continuous (and differentiable) on [0,T] because it is part of the state. By induction, assuming that $\left(x, \ldots x^{(n+i-3)}\right)$ is continuous and differentiable on the closed singular arc, equation (17) proves, since $d(X)$ does not vanish and since $\lambda_{n-i}$ is continuous on $[0, T]$, that $x^{(n+i-2)}$ is continuous and differentiable on the closed singular arc. Moreover, we show by the same induction that $x^{(n+i-2)}$ is a continuous function of the state $X$ and of the adjoint states $\left(\lambda_{n-2}, \ldots, \lambda_{n-i}\right)$. The induction is easily written up to $i=n-1$. This proves that $\left(x, \ldots, x^{(2 n-3)}\right)$ is a set of continuous and differentiable functions on the closed singular arc. Moreover, the derivatives $\left(x^{(n)}, \ldots, x^{(2 n-3)}\right)$ are continuous functions of the state $X$ and the adjoint states $\lambda_{i}$. If the values of this primal and adjoint states are known at the boundary of the singular arc, then they are known at the neighboring regular arcs. These provide boundary conditions for equation (23).

Remark: Once $x$ is computed by solving (23) with suitable boundary conditions, $x$ and its derivatives yield the values of the state, and the values of the adjoint state through (12), (13) and (17). Eventually, the optimal control can be found from $u=x^{(n)}-\sum_{i=1}^{i=n} c_{i} x^{(i-1)}$. While the use of the derivatives of (12) to compute the optimal control in linear problems is not new (see [2]), it has not been brought to the point where the control and trajectories can be effectively obtained by solving an explicitly computed equation.

\section{AN EXAMPLE}

For sake of illustration, we propose to address an example from the literature. A complete exposition of this car trajectory optimization problem can be found in [11]. The dynamics under consideration is

$$
\begin{aligned}
& \frac{d x}{d t}=v \\
& \frac{d v}{d t}=c_{1} u-c_{2} v^{2}-c_{3}-c_{3} \gamma(x)
\end{aligned}
$$

where $u$ is the control which is constrained by $u \in\left[0, u_{\max }\right]$, $x$ is the car position, $v$ its velocity, and $\gamma(x)$ is a known function related to the altitude profile of the specific track the vehicle shall be driven on. To minimize fuel consumption, the cost to be optimized is defined as

$$
J(u)=\int_{0}^{t_{b}} u v d t
$$

where $t_{b}$ is a fixed strictly positive parameter. While the dynamics is slightly more complicated than (9), the cost (25) is much simpler than (10). The computational procedure presented in Section III can be used to compute the control and the trajectory along the singular arcs.

The Hamiltonian is

$$
H=\lambda_{1} v+\lambda_{2}\left(c_{1} u-c_{2} v^{2}-c_{3}-c_{3} \gamma(x)\right)+u v
$$

The adjoint states $\left(\lambda_{1}, \lambda_{2}\right)$ satisfy the following differential equations

$$
\begin{aligned}
& \frac{d \lambda_{1}}{d t}=\lambda_{2} c_{3} \frac{d \gamma}{d x} \\
& \frac{d \lambda_{2}}{d t}=-\lambda_{1}+2 \lambda_{2} c_{2} v-u
\end{aligned}
$$

Here, the switching function is $\frac{\partial H}{\partial u}=\lambda_{2} c_{1}+v$. It is identically zero along the singular arcs. 
The control $u$ does not appear in its first time derivative

$$
\begin{aligned}
\frac{d}{d t}\left(\frac{\partial H}{\partial u}\right) & =c_{1}\left(-\lambda_{1}+2 \lambda_{2} c_{2} v-u\right) \\
& +c_{1} u-c_{2} v^{2}-c_{3}-c_{3} \gamma(x) \\
& =-c_{1} \lambda_{1}+\left[2 c_{1} c_{2} \lambda_{2} v-c_{2} v^{2}\right] \\
& -c_{3}-c_{3} \gamma(x) \\
& =-c_{1} \lambda_{1}-3 c_{2} v^{2}-c_{3}-c_{3} \gamma(x)=0
\end{aligned}
$$

since $\lambda_{2} c_{1}+v=0$ along the singular arcs. Computing the second derivative of the switching function gives

$$
\begin{aligned}
\frac{d^{2}}{d t^{2}}\left(\frac{\partial H}{\partial u}\right) & =-c_{1}\left(\lambda_{2} c_{3} \frac{d \gamma}{d x}\right)+6 c_{2} v \frac{d v}{d t} \\
& -c_{3} \frac{d \gamma}{d x} v \\
& =6 c_{2} v \frac{d v}{d t}=0
\end{aligned}
$$

since $\lambda_{2} c_{1}=-v$. Hence, we have proven that the velocity is constant along singular arcs. Setting (24) to zero gives the optimal control as a feedback along the singular arcs. Then, $\lambda_{1}$ can be computed from (27); $\lambda_{2}$ is given by $\frac{\partial H}{\partial u}=$ $\lambda_{2} c_{1}+v=0$. Recalling $v=\dot{x}$, we finally notice that the states, the adjoint variables, and the control write in terms of $x$ and its derivatives along singular arcs.

\section{Conclusion}

The main contribution of this article is to show that the inversion-inspired elimination procedure used to derive a higher-order form of the stationary conditions of regular optimal control problems can be fruitfully transposed to singular cases of input-constrained optimal control problems. In the example we considered, we were able to derive an interesting property without solving the actual optimal control problem. Had it been desired to compute the optimal trajectories, we would have had to look for the unknown trajectories under the form of a succession of singular and regular arcs. Along these, a limited number of variables (in the presented example, only 1), can be used to represent the numerous unknowns (states, adjoint states and control). Switching times would have been free parameters. Deriving a well suited numerical method is a subject for future research. Addressing MIMO cases is another one.

\section{REFERENCES}

[1] S. K. Agrawal and N. Faiz. A new efficient method for optimization of a class of nonlinear systems without Lagrange multipliers. Journal of Optimization Theory and Applications, 97(1):11-28, 1998.

[2] B. D. O. Anderson and J. B. Moore. Linear Optimal Control. PrenticeHall, 1971.

[3] U. M. Ascher, R. M. M. Mattheij, and R. D. Russell. Numerical solution of boundary value problems for ordinary differential equations. Prentice Hall Series in Computational Mathematics. Prentice Hall, Inc., Englewood Cliffs, NJ, 1988.

[4] U. M. Ascher, R. M. M. Mattheij, and R. D. Russell. Numerical solution of boundary value problems for ordinary differential equations, volume 13 of Classics in Applied Mathematics. Society for Industrial and Applied Mathematics (SIAM), 1995.

[5] R. Bhattacharya. OPTRAGEN: A Matlab toolbox for optimal trajectory generation. In Proc. of the 45th IEEE Conf. on Decision and Control, 2006.
[6] B. Bonnard and M. Chyba. Singular trajectories and their role in control theory, volume 40 of Mathématiques \& applications. SpringerVerlag Berlin Heidelberg New York, 2003.

[7] J. M. Carson III, M. S. Epstein, D. G MacMynowski, and R. M. Murray. Optimal nonlinear guidance with inner-loop feedback for hypersonic re-entry. In Proc. of the 2006 American Control Conference, 2006.

[8] F. Chaplais and N. Petit. Inversion in indirect optimal control. In Proc. of the 7th European Control Conf., 2003.

[9] F. Chaplais and N. Petit. Inversion in indirect optimal control of multivariable systems. ESAIM: Control, Optimisation and Calculus of Variations, 2007 (to appear, preprint available upon request).

[10] P. E. Gill, W. Murray, M. A. Saunders, and M. A. Wright. User's Guide for NPSOL 5.0: A Fortran Package for Nonlinear Programming. Systems Optimization Laboratory, Stanford University, Stanford, CA 94305, 1998.

[11] L. Guzzella. Modeling and control of advanced propulsion systems. In E-COSM. Rencontres scientifiques de l'IFP. New trends in engine control, simulation and modelling, 2006.

[12] C. Hargraves and S. Paris. Direct trajectory optimization using nonlinear programming and collocation. AIAA J. Guidance and Control, 10:338-342, 1987.

[13] A. Isidori. Nonlinear Control Systems. Springer, New York, 2nd edition, 1989.

[14] M. B. Milam. Real-time optimal trajectory generation for constrained systems. PhD thesis, California Institute of Technology, 2003.

[15] M. B. Milam, K. Mushambi, and R. M. Murray. A new computational approach to real-time trajectory generation for constrained mechanical systems. In IEEE Conference on Decision and Control, 2000.

[16] R. M. Murray, J. Hauser, A. Jadbabaie, M. B. Milam, N. Petit, W. B. Dunbar, and R. Franz. Online control customization via optimizationbased control. In T. Samad and G. Balas, editors, Software-Enabled Control, Information technology for dynamical systems, pages 149174. Wiley-Interscience, 2003.

[17] T. Neckel, C. Talbot, and N. Petit. Collocation and inversion for a reentry optimal control problem. In Proc. of the 5th Intern. Conference on Launcher Technology, 2003.

[18] N. Petit, M. B. Milam, and R. M. Murray. Inversion based constrained trajectory optimization. In 5th IFAC Symposium on Nonlinear Control Systems, 2001.

[19] M. C. Steinbach. Optimal motion design using inverse dynamics. Technical report, Konrad-Zuse-Zentrum für Informationstechnik Berlin, 1997.

\section{APPENDIX}

We consider a particular case of the setup presented in Section III. Additionally, we assume that $a$ is a constant, and that $b$ is a linear time invariant function of $X: b(X)=$ $\sum_{i=1}^{i=n} b_{i} x_{i}$. One easily sees that the adjoint dynamics (11) is affine with respect to $\lambda$ and is time invariant. The switching function is $\lambda_{n}+a$. If there is a singular arc, then all the derivatives of $\lambda_{n}$ are zero along the singular arc. Computing these derivatives, we see that the adjoint state is at equilibrium along the singular arc. This also implies $\lambda_{n}+a=0=$ $\lambda_{n} c_{1}+b_{1}$ and, hence, $a c_{1}=b_{1}$. Therefore, if $a c_{1} \neq b_{1}$, there cannot be any singular arc. In this case, since $H$ is constant along the optimal trajectory, $\left(\lambda_{n}+a\right) u$ is differentiable and, hence, $\lambda_{n}+a$ must vanish at the discontinuities of $u$. By contrast, if $a c_{1}=b_{1}$, two (exclusive) cases are possible:

- if the adjoint state is at equilibrium at one point, then it stays there and the optimal control does not appear in any of the derivatives of the switching function

- the adjoint state is never at the equilibrium, and there is no singular arc; as in the case $a c_{1} \neq b_{1}, \lambda_{n}+a$ must vanish at the jumps of $u$. 